\title{
Tailoring NB-IoT for Mass Market Applications: A Mobile Operator's Perspective
}

\author{
Pavel Masek ${ }^{1}$, Martin Stusek ${ }^{1}$, Krystof Zeman ${ }^{1}$, Jiri Hosek ${ }^{1}$, Konstantin Mikhaylov ${ }^{2}$, Sergey Andreev ${ }^{3}$, \\ Yevgeni Koucheryavy ${ }^{3}$, Otto Zeman ${ }^{4}$, Jakub Votapek ${ }^{4}$, and Martin Roubicek ${ }^{4}$ \\ ${ }^{1}$ Department of Telecommunications, Brno University of Technology, Brno, Czech Republic \\ ${ }^{2}$ Centre for Wireless Communications, University of Oulu, Oulu, Finland \\ ${ }^{3}$ Laboratory of Electronics and Communications Engineering, Tampere University of Technology, Tampere, Finland \\ ${ }^{4}$ Vodafone Czech Republic a.s., Prague, Czech Republic \\ $\checkmark$ Contact author's e-mail: masekpavel@vutbr.cz
}

\begin{abstract}
Today, about 6.5 billion "things" are connected to the public space. While this sounds like a lot, still less than $1 \%$ of all the things that are surrounding us and could be connected are actually utilizing this opportunity. Hence, the question is why all these devices are not equipped with wireless connectivity? In some cases, they are either too remote, too inaccessible, or there are simply too many of them to make it economically viable. To mitigate this gap, a promising solution emerges, which has the potential to become an enabler for true mass market IoT. In this paper, we discuss a professional point of view expressed by Vodafone Czech Republic a.s. As Vodafone has been one of the first in Europe to complete a commercial Narrowband IoT (NB-IoT) network roll-out, we build upon this long-term cooperation. First, we discuss the potential of NB-IoT for mass market applications. Then, we offer unique expert findings that reflect the operator's considerations during implementation, testing, measuring, and evaluating this new 3GPP-ratified communications technology. Moreover, we outline our prototype design of the NB-IoT device and discuss the architecture challenges. Also, the configuration required on the side of a mobile operator is detailed to have NBIoT communications ready to take off. Finally, we summarize the results obtained in our lab along with numerous field tests that we run during a long-term cooperation between the research partners.
\end{abstract}

\section{Operator Challenges In LPWANS}

The world is taking a new shape as the next generation of telecommunication systems materializes the vision of hyperconnectivity or, in other words, of an incredibly large number of connections between the diverse elements of the digital world that we see around us. The core that supports the Internet of Things (IoT) is, at the end of the day, exactly that: " $a$ dense heterogeneous network of connections between a multitude of nodes that enables the level of vision, perception, awareness, and, last but not least, control on the online world that was simply not possible even to think about a few years ago" [1].

Presently, mobile network operators (MNOs) have different market requirements that need to be satisfied simultaneously [2]. In the world that now regards connectivity as a commodity, just like electric power, the service is expected to be available whenever it is demanded. Most MNOs have started to consider Machine-to-Machine (M2M) applications as a potential revenue-generating opportunity, and M2M-specific requirements have been introduced by the standardization bodies, such as ETRI, 3GPP, NGMN, etc. At the same time, MNOs are beginning to include in their service portfolios offers that incorporate both connectivity and processing for Business-to-Business (B2B) solutions as a single package.

Still, we are only connecting less than $1 \%$ of what could potentially be connected to the public space. This is because these devices are either too remote, too inaccessible, or there are simply too many of them to make it economically viable. That is why the market for Low Power Wide Area (LPWA) networks, particularly Narrowband-IoT (NB-IoT) [3] and LTE CAT-M1, is heating up these days. More and more MNOs are involved in field trials or have already launched commercial services in this domain. Manufacturers are adding NB-IoT and/or LTE CAT-M1 support to their sensors, modules, and devices [4].

Today, telecom operators have taken a challenging approach to deploy a range of LPWA networking standards for various IoT application needs ${ }^{1}$. However, many are predicting that the market momentum will swing from the first-to-market unlicensed LPWA standards (e.g., LoRaWAN, Sigfox) to the emerging licensed LPWA technologies i.e., 3GPP NB-IoT or LTE CAT-M1, which are envisioned to have a significant share of the LPWA market by 2020 [5].

\section{A. Narrowband IoT Background - Network Perspective}

NB-IoT is a new radio access technology by $3 \mathrm{GPP}$, designed to achieve superior performance w.r.t. the legacy GSM and LTE technologies. Although NB-IoT is not backward compatible with the conventional LTE networks, a significant number of protocols and signaling channels are adapted or modified only marginally. It allows for deploying the NB-IoT systems via a software upgrade of the legacy LTE base stations without the need for new hardware. Generally, NB-IoT simplifies the LTE processes by accommodating the constrained computing power and battery life of NB-IoT devices. This approach is visible in the data transfer signaling as demonstrated in Fig 1. LTE utilizes eight messages to establish a connection and

\footnotetext{
${ }^{1}$ https://www.eetimes.com/document.asp?doc_id=1332811
} 
transfer the data over the network, whereas NB-IoT reduces those signaling overheads to just four messages [6].

Legacy LTE

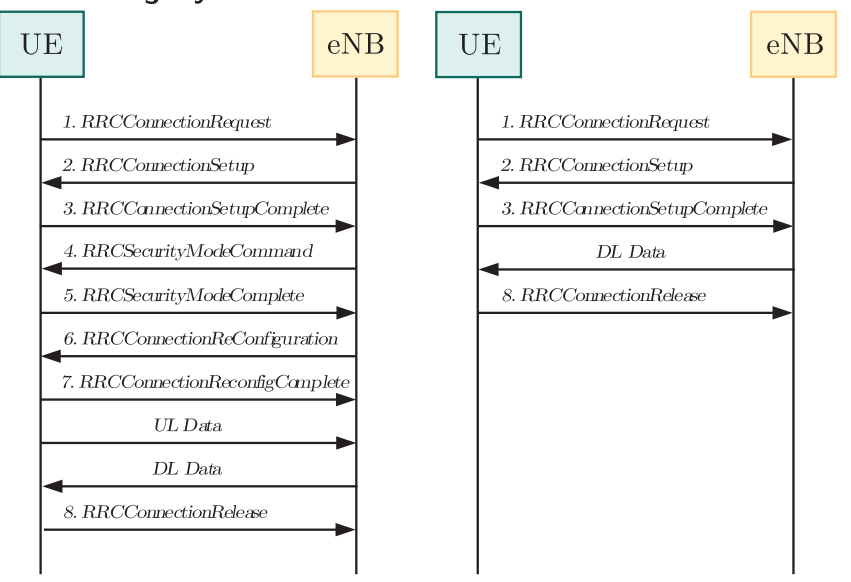

Fig. 1. Comparison between signaling in legacy LTE and NB-IoT.

Another resemblance between NB-IoT and the legacy LTE networks is connected with the frequency domain compatibility. NB-IoT offers three deployment options in the frequency domain: (i) stand-alone, (ii) in-band, and (iii) guard-band implementation. Stand-alone deployment allocates $180 \mathrm{kHz}$ of the GSM carrier by keeping the remaining $20 \mathrm{kHz}$ available as a guard band. In the in-band mode, one or more physical resource blocks (PRBs) are reserved for NB-IoT. This compatibility is possible to reach due to shared numerology - one PRB in the conventional LTE is identical to the bandwidth allocated for the NB-IoT operation. In guard-band regime, the NB-IoT system is implemented within a guard band of the LTE carrier. Therefore, this option is particularly beneficial for telecom operators as it helps save the frequency resources. As the number of allocated radio resources is limited, guardband implementation was chosen for the NB-IoT deployment by Vodafone Czech Republic a.s.

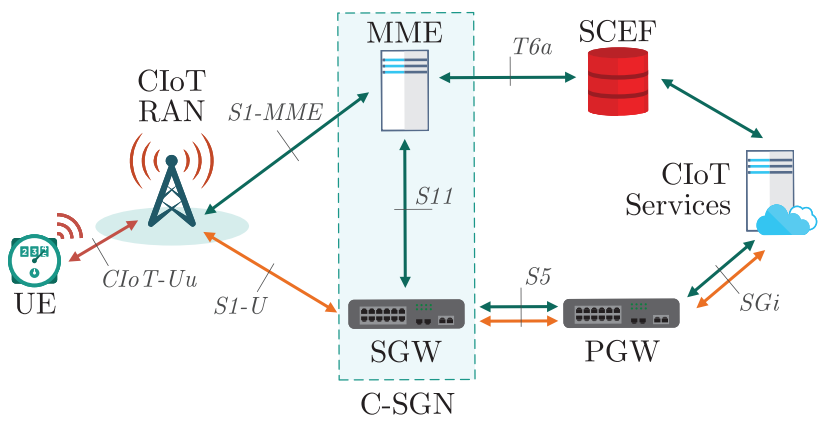

Fig. 2. NB-IoT network architecture.

As mentioned above, from the perspective of network infrastructure, NB-IoT shares its main components with the layout of the current LTE systems. However, two optimizations for the Cellular IoT (CIoT) in the evolved packet system (EPS) were defined - Fig. 2 details Optimized Control Plane CIoT and User Plane CIoT as they are implemented in the NBIoT architecture. Within the Control Plane CIoT optimization, uplink data is transferred from the eNB (CIoT RAN) to the mobility management entity (MME). From this point on, data can be transferred via the serving gateway (SGW) to the packet data network gateway (PGW), or to the service capability exposure function (SCEF). However, the latter is available only for non-IP data packets. Finally, the data from the SCEF or PGW is forwarded to the CIoT.

As bidirectional communication is implemented, data in the downlink direction is routed over the same paths but in a reverse order. This solution does not require a radio bearer to be established, but the data is transmitted over the signaling radio bearer instead. However, this option is feasible only for infrequent data transmissions of small packets. The given description considers the SCEF entity, which is a new node specifically introduced for machine-type data. It is utilized for delivering non-IP packets over the control plane, by providing an abstract interface for the network services. The User Plane (UP) CIoT optimization is designed for data transmission in the same manner as the conventional data traffic i.e., over the radio bearers via SGW and PGW to the CIoT. Hence, it creates certain overheads during a connection setup. However, both IP and non-IP data transfers are supported [7].

\section{B. Main Contributions}

In this work, we report on our unique cooperation with the telecom operator Vodafone Czech Republic a.s., with the goal to empower the NB-IoT mobile network in Czech Republic. First, we discuss the operator's perspective on the NB-IoT technology, continue with NB-IoT market potential, and conclude with a detailed description of the system design choices for massive MTC use-cases. The main contributions of this paper are thus as follows:

- Discussion on internal insights of a mobile operator into the deployment of a commercial NB-IoT service and its tailoring to the mass market applications.

- Design of the NB-IoT enabled evaluation board constructed specifically for the NB-IoT use-cases, which permits a comparison with general and publicly available evaluation kits provided by module manufacturers.

- Results of a 18-months evaluation (since 01/2017) of the NB-IoT technology from the power consumption perspective with a focus on Power Saving Mode (PSM) capabilities in case of battery-limited devices.

- Long-term lab measurements and field tests that verify the extended coverage capability, transmission reliability, and module's behavior in extreme temperature conditions.

\section{NB-IOT MARKET POTENTIAL}

A tremendous growth in the NB-IoT market has motivated analysts to create forecasts showing the expected numbers of connections as well as the revenue potential. Generally, the global IoT market is anticipated to be worth trillions of dollars by 2020 (based on data from ABI Research, Ericsson Mobility report, and SNS Research). The NB-IoT market is a subset of that, and it is important for the MNOs to understand the actual revenue potential in the countries where they operate. 


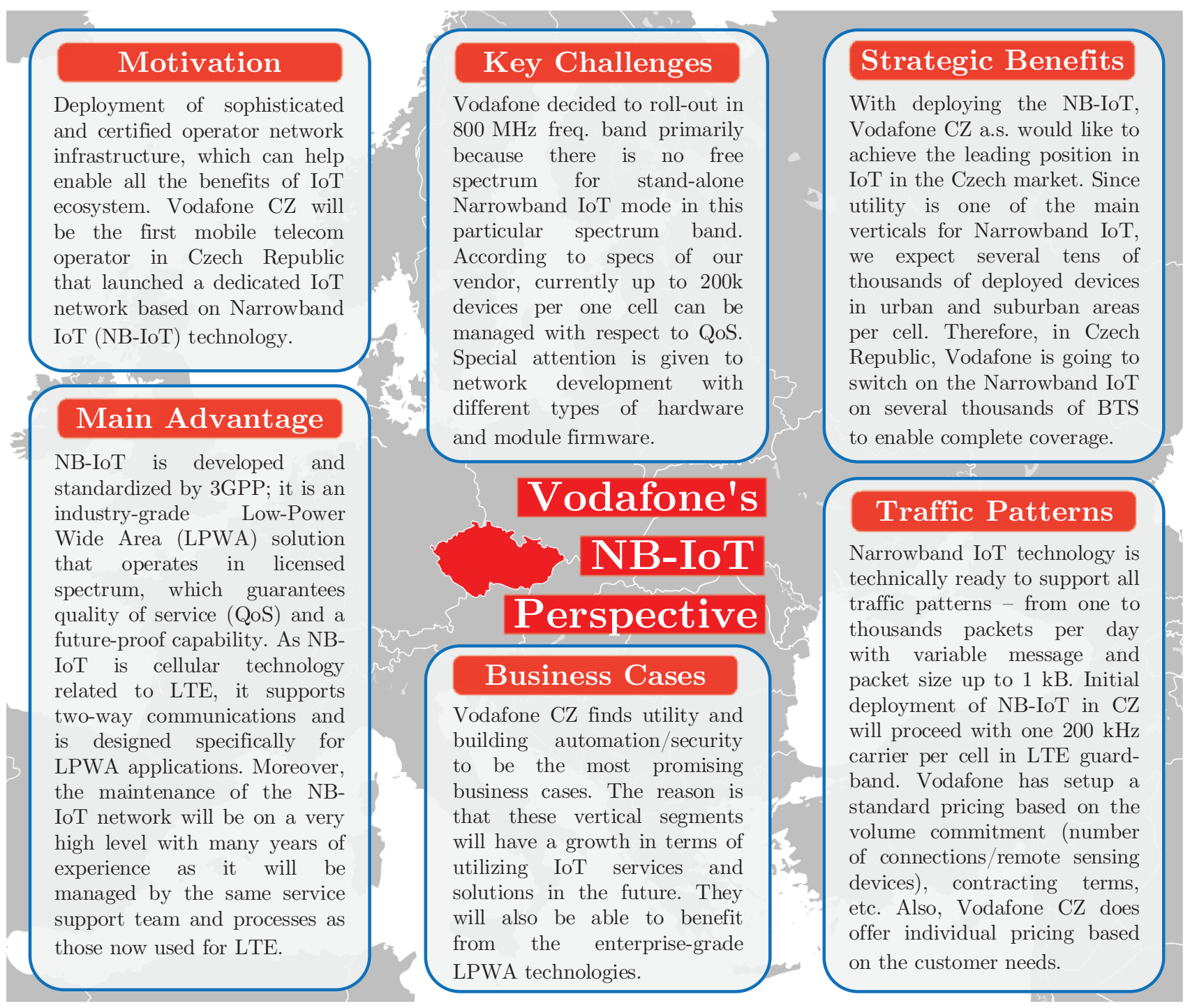

Fig. 4. Vodafone's NB-IoT perspective on massive MTC in Czech Republic. Expert findings conducted in December 2017.

Hence, it is imperative to identify the vertical industries, where NB-IoT can add most value. Owing to the possibility of tight cooperation with the telecom operators, including Vodafone Czech Republic a.s. and A1 Telekom Austria TAG ${ }^{2}$, our forecasts are based on the real use-cases - distinct NB-IoT applications that will be deployed by more than one industry. Of interest are scenarios covering many service categories, such as: (i) smart metering (electricity, gas, and water), (ii) facility management services, (iii) intruder alarms, fire alarms for homes and commercial properties, (iv) connected personal appliances measuring health parameters, (v) tracking of persons, animals, or objects, (vi) smart city infrastructure, such as street lamps or dustbins, and (vii) connected industrial appliances, such as welding machines or air compressors ${ }^{3}$.

\footnotetext{
${ }^{2}$ https://www.vodafone.cz/en/about-vodafone/press-releases/messagedetail/vodafone-letos-rozsviti-celonarodni-sit-pro-nb-iot/

${ }^{3} \mathrm{https} / / / \mathrm{www}$. vodafone.com/business/news-and-insights/whitepaper/iotbarometer
}

By example of Germany, where the Industrie 4.0 initiative (coined by the German Federal Government to optimize industrial production and provide smart manufacturing solutions) was in focus from 2011, the total sum of 1.67 billion USD for five years equals a per-year NB-IoT revenue of 334 million USD. This translates into the revenue uplift of $2.2 \%$ for the existing German telecom operators since the launch of NBIoT services [8]. However, what does this mean for a mobile operator? It creates new MNO monetization opportunities for novel network services to devices and apps, while providing flexibility in how these services are charged for.

As detailed above, the NB-IoT connectivity is a valuable contributor to the operator's business, see Fig. 3. Partnerships with IoT technology providers and alliances, including chipset manufacturers, help the MNOs secure this part of the value chain as we observe today for some of the NB-IoT solutions e.g., smart metering, smart parking, and pet tracking. At the moment, we see that the connectivity platforms already 
move to clouds in many markets where the operators have deployed their IoT services. However, they are more than plain connectivity. MNOs have a chance to go further in the value chain by incorporating further responsibilities, beyond providing pure connectivity.

\begin{tabular}{|c|c|c|}
\hline $\begin{array}{c}\text { Provide } \\
\text { Connectivity }\end{array}$ & $\begin{array}{c}\text { Operate NB-loT } \\
\text { network as a service }\end{array}$ & Move up to value chain \\
\hline $\begin{array}{l}\text { Low ARPC, volume } \\
\text { use cases such as } \\
\text { metering and } \\
\text { tracking. } \\
\text { Connection } \\
\text { management } \\
\text { platform in the } \\
\text { cloud. }\end{array}$ & $\begin{array}{l}\text { ARPC increased due to } \\
\text { an opportunity to } \\
\text { generate service revenue. } \\
\text { Support Big Data } \\
\text { solutions for enhancing } \\
\text { user experience. } \\
\text { Provide full NB-IoT } \\
\text { network functionality in } \\
\text { the cloud. }\end{array}$ & $\begin{array}{l}\text { Competition with OTT and } \\
\text { IT solutions requires fast } \\
\text { and flexible service } \\
\text { integration and delivery. } \\
\text { Much higher ARPC is } \\
\text { possible due to deeper } \\
\text { engagement in service } \\
\text { integration and delivery. }\end{array}$ \\
\hline
\end{tabular}

Fig. 3. Telecom business models for NB-IoT along the value chain.

Consequently, the next step towards an integrated offer would be to incorporate more functionality. This suggests a setup where the telecom operators can offer a fully equipped NB-IoT mobile network as a service in the cloud to the end-toend (E2E) service providers, which are either private or governmental entities, according to the target vertical industries. For the operator, this will create an opportunity to leverage its assets in that domain, such as security, billing, and Big Data. Quality of service (QoS) assurance and Service-Level Agreements (SLA) are common in the telecom world and may be incorporated into the NB-IoT network as a service/business model [9].

Following this thinking further, telecom operators themselves can enter the IoT business as E2E service providers by adding customer management and system integration functionalities on top of their current portfolios. The MNOs as E2E business owners can also outsource a certain part of the E2E domain to their partners - by sharing effort and revenues as well as expand their own experience in the Over-The-Top (OTT) domain. However, competing in the OTT domain is not common for most of today's operators and could be rather challenging.

\section{A. Vodafone's NB-IoT Perspective}

Vodafone Czech Republic a.s. has successfully completed its implementation of the NB-IoT technology and is now testing the NB-IoT system in live operation in partnership with the key industry companies in Czech Republic (jointly with the Department of Telecommunications, Brno University of Technology). Once a number of prototype devices were successfully connected to this NB-IoT setup, the testing was taken further afield to select locations, where the NB-IoT network was established for testing purposes. The technical side of the system infrastructure for the first phase of the NB-IoT pilot was provided by Vodafone in cooperation with Huawei. The NB-IoT network already covers the entire country of Czech Republic (see https://www.vodafone.cz/en/coveragemap/), which has joined Spain and the Netherlands as one of the first European states where Vodafone Group started to offer the emerging IoT communications technology to its clients on a commercial basis.
Vodafone's global IoT platform is a comprehensive solution that comprises advanced connectivity management software integrated with the IoT core network and Vodafone's dedicated global subscriber identity module (SIM), which is not locked to a specific country. The SIM is not geographically localized and connects to the local Vodafone network or roams onto over 500 partner networks anywhere in the world. This ensures the high availability of the IoT services as well as provides predictable costs for connecting IoT devices. The core network is dedicated to the IoT, so that Vodafone has control over its availability, security, and service levels by offering a service that addresses mission-critical IoT use-cases.

Following our productive collaboration, which has started in January 2017, we offer a unified operator's internal perspective on massive MTC over the NB-IoT, see Fig. 4.

\section{System Design For MASSive MTC OVER NB-IoT}

In this section, we discuss our NB-IoT prototype development (in terms of its design, configuration, testing, and measurements), which was completed in cooperation with Vodafone Czech Republic a.s. The solution enables massive MTC and cloudified solutions for IoT, which become the important targets for $5 \mathrm{G}$ and beyond systems.

A practical assessment of the NB-IoT technology in our work includes the design of an evaluation board. This prototype is equipped with a communication module SARA-N210 manufactured by uBlox, which employs the frequency band 20 (832-862 MHz in the uplink and $791-821 \mathrm{MHz}$ in the downlink). The module itself implements the NB-IoT specifications as per Release 13, which features all of the intended energy saving modes i.e., PSM and extended discontinuous reception (eDRX). The remaining capabilities of SARA-N210 include: (i) support for User Datagram Protocol (UDP), (ii) Constrained Application Protocol (CoAP), and (iii) Firmware Over-The-Air (FOTA) update.

Further, the SARA-N210 module promises the data throughput of up to $27.2 \mathrm{kbps}$ in the downlink and $32.25 \mathrm{kbps}$ in the uplink channel (based on the information as of 09/2018). As mentioned above, extended battery life is ensured primarily by PSM, where the current consumption drops below $3 \mu \mathrm{A}$. On the other hand, data transmission introduces peaks of $220 \mathrm{~mA}$ followed by $46 \mathrm{~mA}$ during the message reception and $6 \mathrm{~mA}$ peaks in the idle state ${ }^{4}$.

\section{A. Operator's Implementation}

As discussed previously, Vodafone utilizes guard-band deployment of the NB-IoT carriers. This implementation is efficient in terms of the frequency utilization, thus saves costs as well. The only requirement is the available bandwidth of the LTE carrier of over $5 \mathrm{MHz}$. In the guard-band configuration, $46 \mathrm{dBm}$ is a typical value of the eNB transmit power [6].

In case of NB-IoT, noise significantly impacts the performance by affecting the coding schemes and retransmissions [10]. At this point, the main advantage of the NB-IoT

\footnotetext{
${ }^{4}$ https://www.u-blox.com/en/product/sara-n2-series
} 
over the legacy LTE/LTE-A/GSM is in its extended coverage. This extension was achieved by increasing the maximum coupling loss (MCL) by $20 \mathrm{~dB}$ to $164 \mathrm{~dB}$ [6]. A closer look at the Vodafone's network implementation at the time of the trial implementation (12/2017) reveals that the end devices reside in the roaming mode. This setup eventually influences the communication module settings as the SARA-N210 module is not capable of automatic operator selection in the roaming mode just yet. Hence, this setting was enforced manually in the form of a mobile country code (MCC) and mobile network code (MNC).

\section{B. Design Challenges}

Our first NB-IoT prototype was intentionally designed as a stand-alone battery-powered sensor device. Besides the NBIoT communication module, the prototype was also equipped with a GPS receiver, thus allowing for precise position tracking. However, these design choices brought many challenges during the development process: design of the final product was mostly affected by the power consumption requirements. NB-IoT as compared to a GPS receiver requires a negligible amount of energy, since the module resides in the PSM for most of the time. Due to a substantial battery drain of the active GPS receiver, it is connected via a Metal Oxide Semiconductor Field Effect Transistor (MOSFET), which allows to completely cut-off the power supply and thus saves energy.

As described above, the SARA N210 module was selected for our system design. Unfortunately, the module cannot operate as a stand-alone device. It requires external logic, which is controlling the module via AT commands. The external logic is ensured by the 32-bit Advanced RISC Machine (ARM) microcontroller that communicates with the NB-IoT module via a serial interface. Apart from this, the microcontroller provides two additional serial links, several General-Purpose Input/Output (GPIO) pins, Inter-Integrated Circuit $\left(\mathrm{I}^{2} \mathrm{C}\right)$, Serial Peripheral Interface (SPI), and Controller Area Network (CAN) interfaces, which can be used for communication with external sensors or actuators.

The last part of this system is a charging circuit for built-in LiPol battery as well as serial to Universal Serial Bus (USB) converter that ensures communication with external applications. The design of our NB-IoT prototype is displayed in Fig. 7. To verify the parameters of thus constructed prototype, we conducted long-term measurements in an electromagnetic compatibility (EMC) anechoic chamber as well as in a thermal chamber, both located at Brno University of Technology. The EMC data confirmed that our prototype does not generate undesirable interference. Most importantly, the radio parameters of the SARA N210 module were verified as well. The utilized spectral analyzer confirmed guard-band implementation of the NB-IoT in band 20.

Specifically, the frequencies of $861.7 \mathrm{MHz}$ and $820.7 \mathrm{MHz}$ were utilized in the uplink (see Fig.5) and downlink (see Fig. 6), respectively. Just as importantly, the enhanced sensitivity of the NB-IoT module was confirmed in the EMC chamber. Successful communication was possible to verify even at the

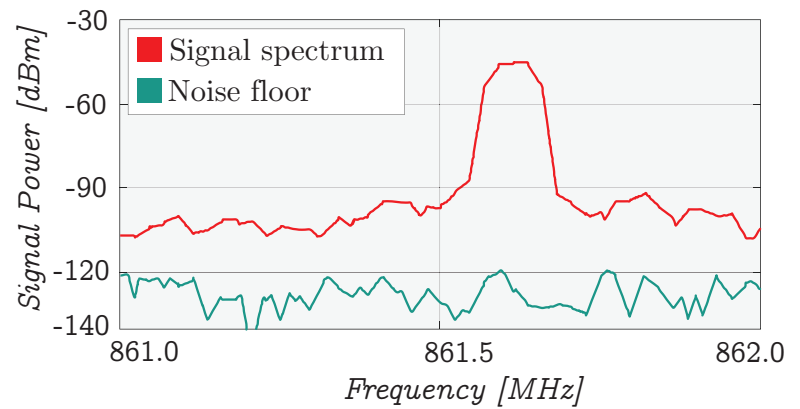

Fig. 5. Vodafone's NB-IoT band 20 utilization for uplink channel (Frequency: 861.7 MHz; EARFCN: 24447).

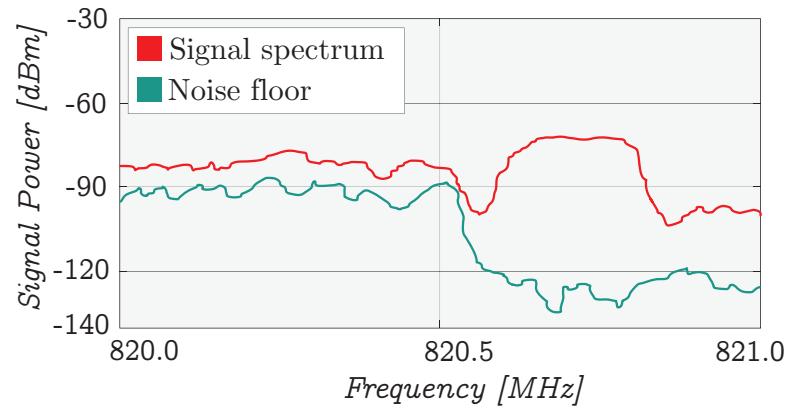

Fig. 6. Vodafone's NB-IoT band 20 utilization for downlink channel (Frequency: $820.7 \mathrm{MHz}$; EARFCN: 6447).

signal levels of under $-135 \mathrm{dBm}$, which is almost $20 \mathrm{~dB}$ gain over the legacy LTE.

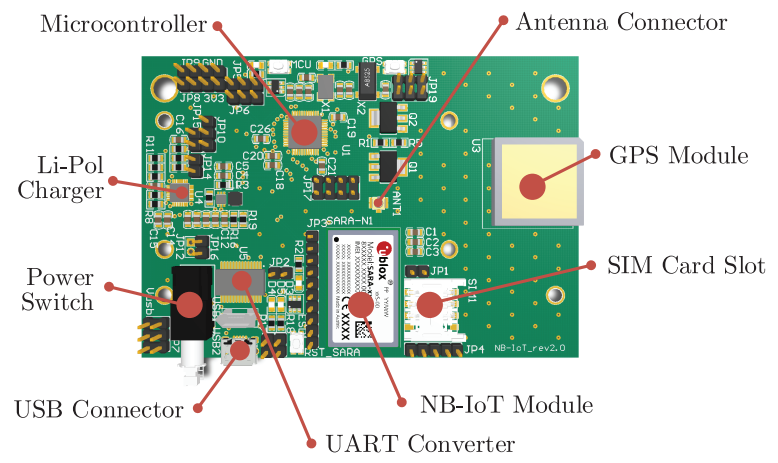

Fig. 7. Developed prototype NB-IoT board.

In our measurements, extreme working conditions were also tested in a thermal chamber. Official operating temperatures advertized by uBlox for the SARA N210 module are within the range of $-40^{\circ} \mathrm{C}$ to $+85^{\circ} \mathrm{C}$. Our measurements verified these assumptions. However, the temperature profoundly impacted the current consumption. At $85^{\circ} \mathrm{C}$, the consumption of the module in PSM raised to $25 \mu \mathrm{A}$, which is more than six times higher as compared to the room temperature of $25^{\circ} \mathrm{C}$ where the current consumption was in the range of 3 to $4 \mu \mathrm{A}$. The rise in consumption at higher temperatures is caused by natural behavior of the semiconductor junction. Hence, temperature growth generates more electron-hole pairs that increase the conductivity by causing a growth in current.

This effect significantly impacts the battery life and has to be considered as the key factor for the lifespan of the LPWAN devices that may operate in such extreme conditions. 


\section{Practical Evaluation}

To evaluate the properties of the NB-IoT technology from the perspectives of power consumption and transmission reliability, we defined two measurement sets as per below.

\section{A. Battery Lifetime Measurements}

First, we focused on the premise of extended battery life provided by the LPWAN technologies. The power consumption of the communication module was measured by utilizing the DC power analyzer Agilent N6705A. To cover all the operating modes of the NB-IoT module, a $120 \mathrm{~s}$ long measurement with a sample period of $0.08 \mathrm{~ms}$ was performed. As depicted in Fig. 8, the measured data allows for distinguishing all of the operating phases, which include: (i) network registration, (ii) reception mode, (iii) release indicator transmission, (iv) $450 \mathrm{~B}$ message transmission, (v) cyclic paging reception, and (vi) transition to the PSM. In the PSM, the consumption decreases to under $4 \mu \mathrm{A}$.

In the aforementioned measurement, the consumption of the NB-IoT module was not optimized - it served as an example demonstrating all the operating modes. In most of the batterypowered devices, the active timer will be shorter than $60 \mathrm{~s}$ to reduce battery drain ${ }^{5}$. Another feature that can save over $90 \%$ of energy is message transmission with release assistance. In this case, the module releases the channel immediately after transmission. Therefore, a new set of measurements with the release assistance enabled was conducted. Here, the active timer (T3324) was set to $2 \mathrm{~s}$, which is the lowest value according to 3GPP TS 24.008 [11]. As shown in Fig. 9, the reduction in power consumption is significant w.r.t. nonoptimized workflow (see Fig. 8). The power optimized version consumes $0.29 \mathrm{~J}$ of energy during a message transmission. On the other hand, the non-optimized setup requires $4.39 \mathrm{~J}$, which is 15 times more.

Consumption difference plays a significant role in the resulting battery life, see Table I, where battery life estimations are given. For this calculation, $3.2 \mathrm{Ah} \mathrm{Li}-\mathrm{SOCl}_{2}$ battery with $3.6 \mathrm{~V}$ nominal voltage was considered ${ }^{6}$. Then the estimated battery life was calculated as follows:

$$
E=E_{T x} \times N_{\text {Reports }}+E_{P S M} \quad[J],
$$

where $E$ is the energy consumed per day, $E_{T x}$ is the energy required for transmission, $N_{\text {Reports }}$ indicates the number of messages per day, and $E_{P S M}$ stands for the energy consumption in PSM. Further, the estimated battery life is:

$$
Y=\left(\frac{C_{B a t}}{E} \times \frac{1}{3600}\right) \times \frac{1}{365},
$$

where $C_{B a t}$ indicates the battery capacity in Watt hours and $E$ stands for the energy consumption per day in Joules.

Surprisingly, even without the power consumption optimization, the resulting battery life is over eleven years in case of two transmitted messages per day. However, with our power

\footnotetext{
${ }^{5}$ For the sake of clarity, we utilized the $60 \mathrm{~s}$ active timer as we intend to highlight all modes clearly.

${ }^{6}$ http://www.titusbattery.com/product/er18505m/
}

optimization, the battery life is prolonged up to 65 years. These results do not however include battery self-discharging, which is about $1 \%$ per year. From the perspectives of long-term deployment, self-discharging plays a crucial role and decreases the resulting lifespan of devices.

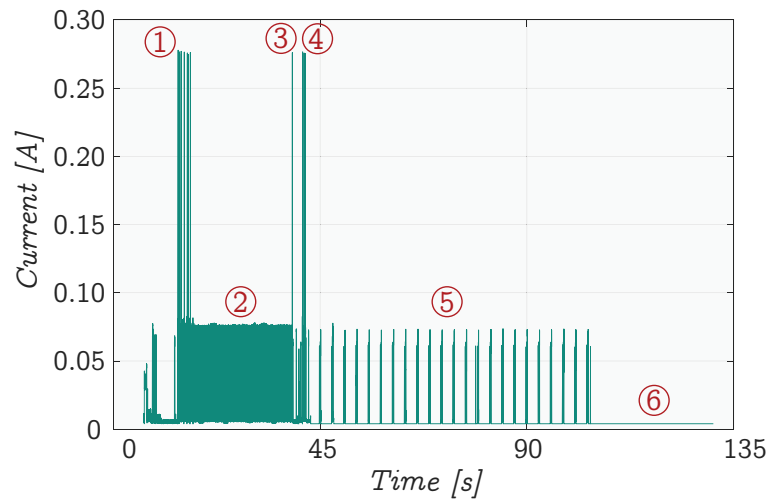

Fig. 8. Power consumption of NB-IoT module in different modes: (1) network registration (2) data reception (3) release indicator transmission (4) message transmission (5) cyclic paging reception (6) power saving mode.

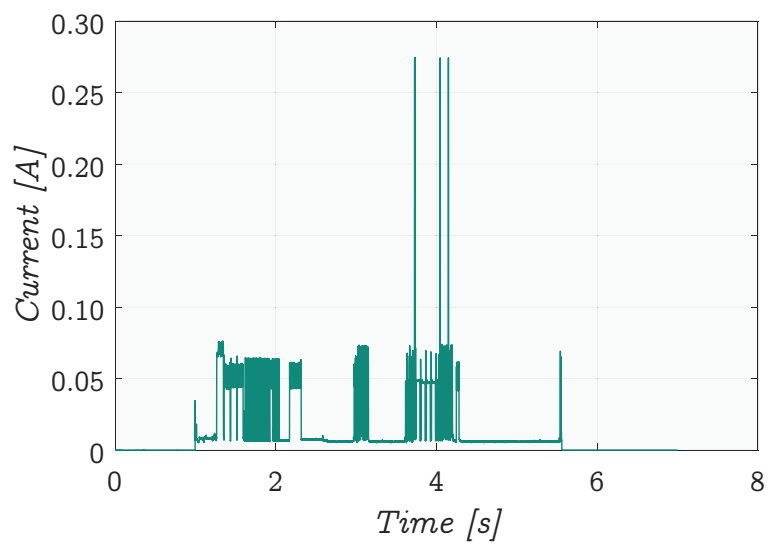

Fig. 9. Power consumption of NB-IoT module with release assistance.

TABLE I

BATTERY LIFE ESTIMATION BASED ON CONDUCTED MEASUREMENTS.

\begin{tabular}{|c|c|c|c|}
\hline Message interval & Message & \multicolumn{2}{|c|}{ Battery life [Years] } \\
\cline { 3 - 4 }$[$ [Hours] & size [B] & Not optimized & Optimized \\
\hline 3 & 450 & 3.13 & 32.55 \\
\hline 6 & 450 & 6.07 & 49.08 \\
\hline 12 & 450 & 11.44 & 65.77 \\
\hline 24 & 450 & 20.52 & 79.25 \\
\hline
\end{tabular}

\section{B. Transmission Reliability}

The second round of our measurements took place in an indoor office environment, where the distance between the eNB and the UE was under $1 \mathrm{~km}$. Its primary purpose was to evaluate the reliability of NB-IoT data transmissions. The actual measurements were conducted during a three-days period to rule out temporary radio interference. The impact of this interference is visible in Fig. 10. At midnight on each day, the signal-to-noise ratio (SNR) significantly decreased down to $0 \mathrm{~dB}$. Despite the degradation in communication quality for the legacy mobile systems (e.g., LTE), the NB-IoT technology is capable of offering reliable data transmissions. 
The significant and repeated drops in the SNR level captured during our measurements (see Fig. 10) are related to specific conditions of the test chamber while the experiments took place there. The conducted measurements confirmed the extended communication range where the messages were transmitted successfully even at such a low value of SNR as $0 \mathrm{~dB}$. According to the $3 \mathrm{GPP}$ recommendations, NB-IoT requires to setup a connection at the SNR level of $-12.6 \mathrm{~dB}$ [12]. Therefore, as said, it was possible to confirm reliable data transmission event at degraded SNR levels.

As already introduced in Table I, 450 B message length was utilized. With 1-minute transmission interval, a total of 1030 messages were transmitted, while the uplink channel indicated $99.9 \%$ reliability. The reliability of the downlink channel followed the uplink statistics as 1022 messages were received successfully i.e., the obtained downlink channel reliability was $99.22 \%$. In a nutshell, both uplink and downlink channels demonstrated above $99 \%$ reliability in our tests.

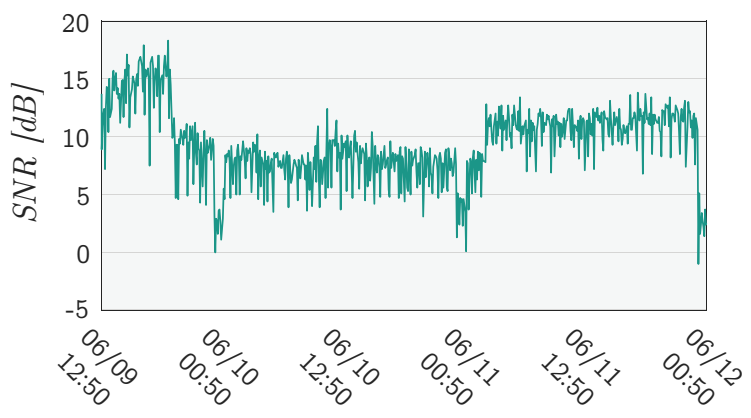

Fig. 10. SNR during NB-IoT transmissions.

\section{Conclusions}

As our vision for the mass utilization of the NB-IoT technology by the forthcoming commercial IoT applications has been confirmed, it becomes clear that the MNOs will have to make further steps to cope with at least the following challenges:

- Technology that is more compatible with the IoT applications will need to be widely available. The recently released standards make a step in that direction, but commercial deployments risk to lag behind.

- MNOs will therefore have to maintain a continuous momentum to evolve their networks and adapt business strategies to successfully manage the challenges posed by other competing technologies, such as LoRaWAN and Sigfox.

- MNOs will also need to have their sales and market strategies adjusted faster than they have been doing until now. The commercial M2M offers will have to be much more common than today.

- Last but not least, security-related aspects as well as capacity planing matters should be taken into account: "mass deployment of inefficient, insecure, or defective IoT devices on the MNOs' networks" may represent a significant threat for the availability and safety of mobile systems, especially in critical situations.
Following the above challenges, it is hard to predict where and when exactly the technology and market evolution will create the true MNO-IoT relationship. Most probably, we will be witnessing an increasing number of scenarios where cellular technologies are employed for specific applications (e.g., services for critical infrastructure). On the other hand, a large proportion of smart devices will utilize other LPWA technologies. To support the statement that the level of the NBIoT capabilities is evolving steadily, the completion of the first European NB-IoT roaming trial was announced by GSMA in July, $2018^{7}$.

In the end, all of the stakeholders will be able to find their niche in the massive MTC market. During our cooperation with Vodafone CZ since January 2017, we have successfully launched and tested the NB-IoT technology in Vodafone's mobile network in Czech Republic. As a summary, the results collected in the lab and field tests are in-line with our initial assumptions - they met our expectations and confirmed the performance parameters of the NB-IoT technology for the real-world deployments in the mass market IoT.

\section{ACKNOWLEDGMENTS}

The described research was supported by the National Sustainability Program under grant LO1401. For the research, infrastructure of the SIX Center was used.

\section{REFERENCES}

[1] U. Raza, P. Kulkarni, and M. Sooriyabandara, "Low power wide area networks: An overview," IEEE Communications Surveys \& Tutorials, vol. 19, no. 2, pp. 855-873, 2017.

[2] J. Hosek, P. Masek, S. Andreev, O. Galinina, A. Ometov, F. Kropfl, W. Wiedermann, and Y. Koucheryavy, "A SyMPHOnY of Integrated IoT Businesses: Closing the Gap between Availability and Adoption," IEEE Communications Magazine, vol. 55, no. 12, pp. 156-164, 2017.

[3] J. Chen, K. Hu, Q. Wang, Y. Sun, Z. Shi, and S. He, "Narrowband Internet of Things: Implementations and applications," IEEE Internet of Things Journal, vol. 4, no. 6, pp. 2309-2314, 2017.

[4] J. Xu, J. Yao, L. Wang, Z. Ming, K. Wu, and L. Chen, "Narrowband Internet of Things: Evolutions, Technologies and Open Issues," IEEE Internet of Things Journal, 2017.

[5] V. Cisco, "Cisco Visual Networking Index: Forecast and Methodology 2016-2021.(2017)," 2017.

[6] O. Liberg, M. Sundberg, E. Wang, J. Bergman, and J. Sachs, Cellular Internet of Things: Technologies, Standards, and Performance. Academic Press, 2017.

[7] J. Schlienz, D. Raddino, "Narrowband Internet of Things," tech. rep., Rhode \& Schwarz GmbH \& Co. KG, 2016.

[8] A. Nordrum et al., "Popular Internet of Things forecast of 50 billion devices by 2020 is outdated," IEEE Spectrum, vol. 18, 2016.

[9] G. A. Akpakwu, B. J. Silva, G. P. Hancke, and A. M. Abu-Mahfouz, "A survey on 5G networks for the Internet of Things: Communication technologies and challenges," IEEE Access, vol. 6, pp. 3619-3647, 2018.

[10] J. Xu, J. Yao, L. Wang, Z. Ming, K. Wu, and L. Chen, "Narrowband Internet of Things: Evolutions, Technologies and Open Issues," IEEE Internet of Things Journal, pp. 1-1, 2017.

[11] ETSI TS 124 008: "Digital cellular telecommunications system (Phase 2+) (GSM); Universal Mobile Telecommunications System (UMTS); LTE; Mobile radio interface Layer 3 specification; Core network protocols; Stage 3 ”, Ver. 13.5.0, 2016-04.

[12] A. Ali and W. Hamouda, "On the Cell Search and Initial Synchronization for NB-IoT LTE Systems," IEEE Communications Letters, vol. 21, pp. 1843-1846, Aug 2017.

\footnotetext{
${ }^{7}$ https://www.gsma.com/newsroom/press-release/gsma-announcescompletion-of-first-european-nb-iot-roaming-trial/
} 